\title{
Informationen zum Qualitätssicherungsregister Interventionelle Radiologie der Deutschen Gesellschaft für Interventionelle Radiologie und minimalinvasive Therapie (DeGIR)
}

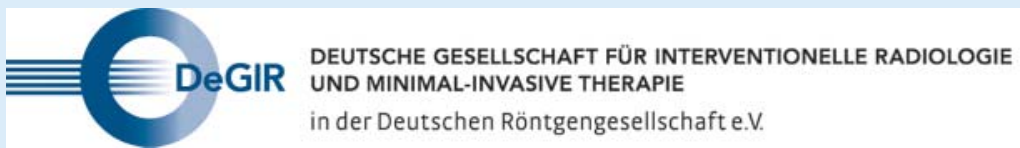

in der Deutschen Röntgengesellschaft e.V.
Seit dem Jahr 2005 dokumentieren deutschlandweit (sowie in Österreich und in der Schweiz) radiologische Einrichtungen ihre Eingriffe (Module A-D) im Qualitätsregister Interventionelle Radiologie der Deutschen Gesellschaft für Interventionelle Radiologie und minimalinvasive Therapie (DeGIR). Die Deutsche Gesellschaft für Neuroradiologie (DGNR) schloss sich der Dokumentation mit den neuroradiologischen Interventionen (Module E und F) im Jahre 2012 an.

Seit dem 01.01.2018 ist das webbasierte Register bei dem Berliner Spezialisten für WebSoftwarelösungen (SaaS) im Gesundheitswesen, samedi ${ }^{\circledR}$, beheimatet. Dieser Anbieterwechsel stellt einen deutlichen Fortschritt dar, da wir nun ein weitaus flexibleres System an der Hand haben, welches wir an unsere Bedürfnisse anpassen können. Zur Dokumentation der durchgeführten Eingriffe und deren Ergebnisqualität nutzen aktuell über 280 Kliniken das Qualitätssicherungsregister.

Eine Verwendung des Datenmaterials für wissenschaftliche Publikationen und berufspolitische Aussagen - etwa zur Versorgungsdichte, erbrachten Fallzahlen bei bestimmten Indikationen und Zentren-Bildung erfolgte bislang nur sporadisch. Ein aktuelles Publikationsprojekt zum Thema Thrombektomie-Versorgung in Deutschland (Autoren: Berlis, A., Morhard, D, Weber, W.) stellt die Eingabezahlen zur mechanischen Re-Kanalisation beim akuten ischämischen Schlaganfall dar. Es handelt sich hierbei um eine Folgepublikation der bereits 2017 erschienenen Darstellung der dokumentierten Interventionszahlen von 2015.

Publikationen dieser Art sind wichtig, weil sie die Schlagkraft der interventionellen (Neuro-)Radiologie gegenüber an- deren interventionell tätigen Fächern der Medizin unter Beweis stellen.

Bei Veröffentlichungen der dokumentierten Daten, welche über die vertraglich geregelten Auswertungen hinausgehen, sind wir auf Ihr Einverständnis angewiesen. Wir möchten Sie daher bitten, Ihre Fallzahlen auch in Zukunft im Sinne der interventionellen (Neuro-) Radiologie für Publikationsprojekte dieser Art zur Verfügung zu stellen. Die Verwendung der Daten wird selbstverständlich immer unter datenschutzrechtlichen Maßgaben und nur nach explizit erteilter Zustimmung der verantwortlichen Personen erfolgen.

Es ist uns und den Mitgliedern der Software-Lenkungsgruppe bewusst, dass es Abweichungen bezüglich der von Ihnen eingegebenen Daten und den tatsächlich codierten Fällen Ihrer Kliniken gibt. Diese Abweichungen liegen im Eingabeverhalten der jeweiligen Häuser begründet und sind mit Blick auf die Dokumentationsbelastung der einzelnen Abteilungen oftmals nachvollziehbar. Dennoch möchten wir Sie dazu aufrufen, im Sinne der Leistungsdarstellungen der interventionellen (Neuro-) Radiologie möglichst viele Eingriffe im Qualitätssicherungsregister zu dokumentieren.

Auch in Zukunft werden Sie immer bis zum 28. Februar des Folgejahres Gelegenheit haben, Ihre Interventionen für das abgelaufene Jahr zu dokumentieren. Die Geschäftsstelle der DeGIR wird Sie hierüber zu gegebener Zeit und in gebotener Detaillierung informieren.

Die wissenschaftlich fundierte Auswertung und Nutzung der dokumentierten Daten ist ausbaufähig und soll im Sinne der interventionellen (Neuro-) Radiologie künftig weiter vorangetrieben werden. Mit der Lenkungs- gruppe „Wissenschaft und Forschung“ hat sich auf dem IROS 2018 eine Initiative gebildet, welche der Software-Lenkungsgruppe dabei zur Seite stehen wird. Mit dem neuen Stroke-Formular im Modul E sollen künftig auch genauere Auswertungen bezüglich der Schlaganfallbehandlung möglich sein. Bitte unterstützen Sie auch diese Projekte durch Ihr ungebrochenes Dokumentationsverhalten. Nur auf Basis einer großen und qualitativ hochwertigen Datenmenge lassen sich studienrelevante Aussagen treffen und öffentliche Aufmerksamkeit generieren.

Abschließend möchten wir noch einmal ausdrücklich allen Mitarbeiterinnen und Mitarbeitern der Kliniken danken, die sich seit vielen Jahren an der Dokumentation im Qualitätssicherungsregister beteiligen. Wir versichern Ihnen, dass die SoftwareLenkungsgruppe auch in Zukunft in enger Abstimmung mit den Vorständen der DeGIR und der DGNR und im Rahmen unserer finanziellen Möglichkeiten an der Weiterentwicklung und Verbesserung des Registers arbeiten wird.

Mit kollegialen Grüßen

Prof. Dr. med. Peter Reimer (Karlsruhe) Vorstandsvorsitzender der DeGIR

Prof. Dr. med. Arnd Dörfler (Erlangen) Präsident der DGNR

Prof. Dr. med. Arno Bücker (Homburg) Leiter der Software-Lenkungsgruppe in der DeGIR

Prof. Dr. med. Werner Weber (Bochum) Mitglied der Software-Lenkungsgruppe für die DGNR

29.06 .2018 